\title{
Combinação dos Tratamentos Biológico e Químico de Semente de Milho
}

\author{
Wilmar Cório da Luz* \\ Embrapa Trigo, Cx. Postal 451, CEP 99001-970, Passo Fundo, RS, Brasil, e-mail: wilmar@cnpt.embrapa.br
}

(Aceito para publicação em 18/10/2002)

LUZ, W.C. da. Combinação dos tratamentos biológico e químico de semente de milho. Fitopatologia Brasileira 28:037-040. 2003.

\section{RESUMO}

A integração de métodos de proteção de semente de milho (Zea mays) poderá reduzir consideravelmente o uso de pesticidas nessa cultura, melhorando a eficiência de controle e diminuindo a poluição do ambiente. O objetivo do presente trabalho foi determinar o efeito da combinação dos controles biológico e químico no tratamento de semente de milho. Como controle biológico, foi usado o bioprotetor Embr. 144 e, como controle químico, foi empregado fludioxonil + metalaxyl M. Dois experimentos de laboratório e dois de campo foram conduzidos na município de Passo Fundo, RS. Os tratamentos que continham o bioprotetor Paenibacillus macerans (Embr. 144) ou o fungicida fludioxonil + metalaxyl-M, ou ambos, reduziram significativamente o nível de infecção dos patógenos nas sementes, destacando-se os tratamentos que continham ambos, os quais eliminaram por completo todos os patógenos da semente de milho, demonstrando que a interação entre o bioprotetor e o fungicida apresentou benefícios. Mesmo quando as doses do bioprotetor e do fungicida no tratamento integrado foram reduzidas à metade, o nível de controle dos patógenos de milho foi superior ao nível de controle do bioprotetor e do fungicida isoladamente, com as doses normais. Todos os tratamentos aumentaram significativamente a germinação de sementes e o rendimento de grãos de milho em relação à testemunha, entretanto, os efeitos foram superiores quando houve a combinação dos tratamentos do bioprotetor com o fungicida. A combinação do bioagente P. macerans com o produto fludioxonil + metalaxyl-M poderá ter importante impacto na redução do uso de fungicidas, para alcançar a agricultura sustentável e proteger o ambiente.

Palavras-chave adicionais: controle biológico, tratamento de semente, patógenos, milho, Zea mays.

\section{ABSTRACT}

Biological and chemical treatment combinations for corn seeds The integration of methods for corn (Zea mays) seed protection may substantially reduce the use of pesticides in this crop, improving control efficiency and diminishing environmental pollution. Experiments were carried out under laboratory and field conditions in Passo Fundo, RS, Brazil, aimed at evaluating the effects of combining biological and chemical seed treatments for corn. The bioprotector used was Paenibacillus macerans (Embr. 144) and the chemical was a mixture of fludioxonil + metalaxyl-M. P. macerans, which significantly improved germination and grain yield of corn, showing increases similar to fludioxonil + metalaxyl-M. Seed germination and grain yield were significantly greater when $P$. macerans was applied in combination with fludioxonil + metalaxyl$\mathrm{M}$, even if both treatment rates were reduced to half. The combination of bioagent $P$. macerans with the fungicide fludioxonil + metalaxyl-M may have an important impact in reducing the use of fungicides, in achieving the sustainable agriculture and protecting the environment.

\section{INTRODUÇÃO}

As sementes na cultura do milho (Zea mays L.) no Brasil são atacadas por vários fungos. As perdas em conseqüência desses patógenos, embora não determinadas, são comumente reconhecidas. Em conseqüência, o tratamento de semente de milho com fungicidas é uma prática rotineira contra essas doenças.

Vários fungicidas têm sido investigados com resultados de eficiência contra os principais patógenos de milho (Luz 1996b,c,d,e, 1997a,b, 1998a,b, Luz \& Pereira 1998). Há necessidade de emprego de produtos biológicos, que sirvam como alternativa, em combinação com o produto químico, para melhorar o controle dos principais patógenos e aumentar o rendimento de grãos da cultura.

$\overline{{ }^{*} \text { Bolsista do CNPq. }}$
A microbiolização de semente tem sido realizada para controle de vários patógenos e também como promotora de germinação e crescimento de plantas de milho (Chang \& Kommedahl, 1968; Callan et al.1990,1991; Luz 1996a,b; Luz et al. 1997a,b; Luz 2001a). Paenibacillus macerans (Embr. 144) é um bioprotetor isolado no Brasil e apresenta um amplo espectro de ação contra fungos de cereais que são transmitidos pelas sementes e pelo solo (Luz, 1996a,b,; 2001,a; Luz et al. 1997a,b). Possui propriedades antagônicas documentadas sobre Fusarium graminearum Schw., Drechslera triticirepentis (Died.) Shoem., Bipolaris sorokiniana (Sacc. in Sorok.) Shoem. e Stagonospora nodorum (Berk.) Cast. \& Germ. (Luz, 1996a,b; 2001,a; Luz et al 1997a,b) em trigo (Triticum aestivum L.) e sobre F. graminearum, Diplodia maydis (Beek) Sacc. e F. moniliforme Sheldon em milho (Luz, 1996a; Luz, 2001a; LUZ et al. 1997a,b)

Os objetivos do presente estudo foram: 1) verificar o 
efeito do tratamento de semente com o bioprotetor $P$. macerans (Embr. 144) sobre os fungos patogênicos de semente de milho; 2) estudar o efeito do fungicida fludioxonil + metalaxyl-M (Maxim XL) sobre esses patógenos; e 3) determinar o efeito da interação dos tratamentos biológico e químico na semente de milho.

\section{MATERIAL E MÉTODOS}

Os experimentos foram realizados em Passo Fundo, RS (Embrapa Trigo) em 1999 e 2000. As sementes de milho do híbrido BR 5202, suscetível aos patógenos da semente usada neste estudo, foram obtidas da Embrapa. Os tratamentos foram: Testemunha; P. macerans - Embr. $144(11,0 \mathrm{~g} / 100 \mathrm{~kg}$ de sementes); P. macerans - Embr. 144 (11,0 g/100 kg de semente $)+$ fludioxonil $(2,5 \%$ i.a. $)+$ metalaxyl-M $(1,0 \%$ i.a.) $150 \mathrm{ml}$ do produto comercial/100 kg de semente]; $P$. macerans - Embr. 144 (11,0 g/100 kg de semente) + [fludioxonil (2,5\% i.a.) + metalaxyl-M (1,0\% i.a.) ] (75 ml/ $100 \mathrm{~kg}$ de semente); [fludioxonil (2,5\% i.a) + metalaxyl-M (1,0\% i.a.)] (150 ml do produto comercial/100 kg de semente); P. macerans - Embr. $144(5,5 \mathrm{~g} / 100 \mathrm{~kg} / \mathrm{semente})+$ [fludioxonil $(2,5 \%$ i.a $)+$ metalaxyl-M (1,0\% i.a.)] $(75 \mathrm{ml} / 100 \mathrm{~kg}$ de semente); P.macerans - Embr.114 (11,0 g/100 kg/semente) + [fludioxonil (2,5\% i.a) + metalaxyl-M (1,0\% i.a.) ] (150 ml/ $100 \mathrm{~kg}$ de semente).

No experimento de laboratório, cada tratamento foi constituído de 100 sementes distribuídas igualmente por 20 placas, repetido quatro vezes, e colocado sob luz negra com fotoperíodo de $12 \mathrm{~h}$ à temperatura de $24 \pm 2{ }^{\circ} \mathrm{C}$. O arranjo experimental foi blocos inteiramente casualizados. A presença dos fungos patogênicos foi determinada cinco dias após o plaqueamento. Os dados foram expressos em percentagem referente à incidência de cada patógeno nas sementes.

Nos experimentos de campo, as parcelas foram compostas de quatro fileiras de $5 \mathrm{~m}$ cada uma. $\mathrm{O}$ espaçamento entre fileiras foi de $1 \mathrm{~m}$, e entre sementes, de $20 \mathrm{~cm}$. O delineamento experimental foi de blocos ao acaso, com quatro repetições. A emergência de sementes foi avaliada 30 dias após a semeadura. Na maturação, as espigas foram colhidas, os grãos trilhados e pesados com umidade de $13 \%$. Os dados dos tratamentos foram submetidos à análise de variância, e as médias foram comparadas usando-se o teste de LSD de Fisher, ao nível de 0,05 de probabilidade.

\section{RESULTADOS E DISCUSSÃO}

Nos experimentos de laboratório, as sementes de milho apresentavam-se severamente infetadas com os fungos $F$. graminearum, F. moniliforme, D. maydis e Aspergillus spp., principalmente A. glaucus (Link ex Gray) e A. flavus (Lk. ex. Fr) (Tabela 1). A incidência de patógenos em 1999 foi superior à de 2000, devido às condições ambientais da safra anterior.

Os tratamentos que continham o bioprotetor $P$. macerans (Embr. 144) ou o fungicida fludioxonil + metalaxylM, ou ambos, reduziram significativamente o nível de infecção dos patógenos nas sementes, destacando-se os tratamentos que continham ambos, os quais eliminaram todos os patógenos da semente de milho, demonstrando que a interação entre o bioprotetor e a associação fungicida apresentou benefícios. Mesmo quando a dose do bioprotetor e da associação fungicida, no tratamento integrado, foi reduzida à metade, o nível de controle dos patógenos de milho foi superior ao nível de controle do bioprotetor e da associação fungicida isoladamente com as doses normais.

Todos os tratamentos aumentaram significativamente a germinação de sementes e o rendimento de grãos de milho, em relação à testemunha (Tabela 2), entretanto os efeitos foram superiores quando houve a combinação dos tratamentos do

TABELA 1 - Efeito da associação do tratamento químico e biológico de sementes no controle de patógenos de milho (Zea mays). Embrapa BR 5202

\begin{tabular}{|c|c|c|c|c|c|c|c|c|c|c|}
\hline \multirow{3}{*}{$\begin{array}{l}\text { Tratamento e dose do produto comercial } \\
\text { por } 100 \mathrm{~kg} \text { de sementes }\end{array}$} & \multicolumn{10}{|c|}{ Fungos em semente de milho (\%)* } \\
\hline & \multicolumn{2}{|c|}{$\begin{array}{l}\text { Diplodia } \\
\text { maydis }\end{array}$} & \multicolumn{2}{|c|}{$\begin{array}{c}\text { Fusarium } \\
\text { moniliforme }\end{array}$} & \multicolumn{2}{|c|}{$\begin{array}{c}\text { Fusarium } \\
\text { graminearum }\end{array}$} & \multicolumn{2}{|c|}{$\begin{array}{l}\text { Aspergillus } \\
\text { spp. }\end{array}$} & \multicolumn{2}{|c|}{$\begin{array}{l}\text { Penicillium } \\
\text { spp. }\end{array}$} \\
\hline & 1999 & 2000 & 1999 & 2000 & 1999 & 2000 & 1999 & 2000 & 1999 & 2000 \\
\hline Testemunha & $25 \mathrm{c}$ & $11 \mathrm{c}$ & $24 \mathrm{c}$ & $15 \mathrm{c}$ & $4 \mathrm{~b}$ & $3 \mathrm{~b}$ & $23 \mathrm{c}$ & $14 \mathrm{c}$ & $32 \mathrm{c}$ & $17 \mathrm{c}$ \\
\hline Paenibacillus macerans (Embr. 144), 11,0 g & $4 \mathrm{~b}$ & $3 \mathrm{~b}$ & $8 \mathrm{~b}$ & $5 \mathrm{~b}$ & $0 \mathrm{a}$ & $0 \mathrm{a}$ & $4 \mathrm{~b}$ & $3 \mathrm{~b}$ & $5 \mathrm{~b}$ & $4 \mathrm{~b}$ \\
\hline $\begin{array}{l}\text { P. macerans }(\text { Embr. 144), }(11,0 \mathrm{~g})+\text { [fludioxonil }+ \\
\quad \text { metalaxyl-M }(75 \mathrm{ml})]\end{array}$ & $0 \mathrm{a}$ & $0 \mathrm{a}$ & $0 \mathrm{a}$ & $0 \mathrm{a}$ & $0 \mathrm{a}$ & $0 \mathrm{a}$ & $0 \mathrm{a}$ & $0 \mathrm{a}$ & $0 \mathrm{a}$ & $0 \mathrm{a}$ \\
\hline $\begin{array}{l}\text { P. macerans (Embr. 144), (11,0 g) + [fludioxonil + } \\
\text { metalaxyl-M }(150 \mathrm{ml})]\end{array}$ & $0 \mathrm{a}$ & $0 \mathrm{a}$ & $0 \mathrm{a}$ & $0 \mathrm{a}$ & $0 \mathrm{a}$ & $0 \mathrm{a}$ & $0 \mathrm{a}$ & $0 \mathrm{a}$ & $0 \mathrm{a}$ & $0 \mathrm{a}$ \\
\hline Fludioxonil + metalaxyl-M (150 ml) & $6 \mathrm{~b}$ & $4 \mathrm{~b}$ & $7 \mathrm{~b}$ & $3 \mathrm{~b}$ & $0 \mathrm{a}$ & $0 \mathrm{a}$ & $5 \mathrm{~b}$ & $3 \mathrm{~b}$ & $3 \mathrm{~b}$ & $2 b$ \\
\hline $\begin{array}{l}\text { P. macerans (Embr. 144), }(5,5 \mathrm{~g})+\text { [fludioxonil }+ \\
\text { metalaxyl-M }(75 \mathrm{ml})]\end{array}$ & $0 \mathrm{a}$ & $0 \mathrm{a}$ & $0 \mathrm{a}$ & $0 \mathrm{a}$ & $0 \mathrm{a}$ & $0 \mathrm{a}$ & $0 \mathrm{a}$ & $0 \mathrm{a}$ & $0 \mathrm{a}$ & $0 \mathrm{a}$ \\
\hline C.V. \% & 3,1 & 4,9 & 3,7 & 5,3 & 2,2 & 2,0 & 3,5 & 4,4 & 3,6 & 3,9 \\
\hline
\end{tabular}

*Média de quatro repetições. Dados seguidos por letras iguais, nas colunas, não diferem entre si, de acordo com o teste de LSD de Fisher, a p=0,05. 
TABELA 2 - Efeito da associação bioprotetor Embr. 144 com fungicidas fludioxonil + metalaxyl-M empregados no tratamento de sementes sobre a germinação das sementes sobre o complexo do enfezamento e o rendimento de grãos do milho (Zea mays)

\begin{tabular}{|c|c|c|c|c|}
\hline \multirow{2}{*}{ Tratamento* } & \multicolumn{2}{|c|}{ Germinação**(\%) } & \multicolumn{2}{|c|}{ Rendimento** $(\mathrm{kg} / \mathrm{ha})$} \\
\hline & 1999 & 2000 & 1999 & 2000 \\
\hline Testemunha & $77 \mathrm{c}$ & $78 \mathrm{c}$ & $5.618 \mathrm{c}$ & $6.723 \mathrm{c}$ \\
\hline Paenibacillus macerans (Embr. 144), 11,0 g & $86 \mathrm{~d}$ & $89 \mathrm{~b}$ & $6.124 b$ & $7.329 \mathrm{~b}$ \\
\hline P. macerans (Embr. 144), $(11,0 \mathrm{~g})+[\text { fludioxonil + metalaxyl-M }(75 \mathrm{ml})]^{1}$ & $93 \mathrm{a}$ & $96 \mathrm{a}$ & $6.626 \mathrm{a}$ & $7.811 \mathrm{a}$ \\
\hline P. macerans (Embr. 144), (11,0 g)+ [fludioxonil + metalaxyl-M (150ml)] & 94 a & $97 \mathrm{a}$ & $6.633 \mathrm{a}$ & $7.841 \mathrm{a}$ \\
\hline Fludioxonil + metalaxyl-M (150 ml) & $87 \mathrm{~b}$ & $88 \mathrm{~b}$ & $6.119 \mathrm{~b}$ & $7.412 \mathrm{~b}$ \\
\hline P. macerans (Embr.144), (5,5 g) + [fludioxonil + metalaxyl-M (75 ml)] & $92 \mathrm{a}$ & 95 a & $6.627 \mathrm{a}$ & $7.837 \mathrm{a}$ \\
\hline C.V. $\%$ & 7,9 & 10,4 & 8,3 & 12,7 \\
\hline
\end{tabular}

* Dose do produto comercial para $100 \mathrm{~kg}$ de sementes

** Média de quatro repetições. Dados seguidos por letras iguais, nas colunas, não diferem entre si, de acordo com o teste de LSD de Fisher, a p = 0,05.

bioprotetor com o fungicida em quaisquer dosagens testadas. Nenhum sintoma de fitotoxidez foi observado no experimento. A ação desse bioprotetor está ligada ao controle de fungos patogênicos de semente que causam má germinação, início ou aumento de inóculo de patógenos que facilitam o desenvolvimento de doenças em sementes, em plântulas e em planta adulta, reduzindo o rendimento de grãos de milho. Mecanismos de ação, tais como indução de resistência, fito-hormônios, mineralização de nutrientes e outros, podem estar envolvidos (Kloepper 1991; Boronin et al., 1993; Liu et al.; 1995 a,b; Luz, 1996a). Esses mecanismos de ação também podem estar agindo em interação com o controle direto de patógenos por parte dos produtos empregados nos tratamentos.

O tratamento químico de semente de milho com fungicidas, apesar de ter sido amplamente estudado (Luz, 1996a, b; 1997b, c; 1998d; Luz \& Pereira 1998), não tem sido avaliado em combinação com o controle biológico. Por esse motivo, os resultados deste estudo apresentam-se inovadores e promissores, e demonstram que o bioprotetor $P$. macerans (Embr. 144), em combinação com fludioxonil + metalaxyl-M, é uma alternativa ao tratamento convencional de sementes de milho. A combinação desse bioagente com o produto fludioxonil + metalaxyl-M poderá ter importante impacto na redução do uso de fungicidas, para alcançar a agricultura sustentável e proteger o ambiente.

\section{REFERÊNCIAS BIBLIOGRÁFICAS}

BORONIN, A.M., KOCHETKOV, V.V., DUBEIKOVSKI, A.N. \& MORDUKHOVA, E.A. Biological control of soilborne plant, pathogens by PGPR Pseudomonas isolated in Russia. Abstract, $7^{\circ}$ International Congress of Plant Pathology, Montreal, Canada, International Society of Plant Pathology. 1993. p.276.

CALLAN, N.W., MATHRE, D.E. \& MILLER, J.B. Bio-priming seed treatment for biological control of Pythium ultimum preemergence damping-off in sh 2 sweet corn. Plant Disease 74:368372. 1990.

CALlAn, N.W., MAThre, D.E. \& MILlER, J.B. Field performance of sweet corn seed bio-primed and coated with
Pseudomonas fluorescens AB254. HortScience 26:1163-1169. 1991.

CHANG, I. \& KOMMEDAHL, T. Biological control of seedling blight of corn by coating kernels with antagonistic microorganisms. Phytopathology 58:1395-1401. 1968.

KLOEPPER, J.W. Plant growth-promoting rhizobacteria as biological control agents of soilborne diseases. In: Bay-Peterson, J. (Ed.).The biological control of plant diseases. Taiwan, Food and Fertilizer Technological Center. 1991. pp.142-152.

LIU, L., KLOEPPER, J.W. \& TUZUN, S. Induction of systemic resistance in cucumber against bacterial angular leaf spot by plant growth-promoting rhizobacteria. Phytopathology 85:843-847. 1995a.

LIU, L., KLOEPPER, J.W. \& TUZUN, S. Induction of systemic resistance in cucumber against Fusarium wilt by plant growthpromoting rhizobacteria. Phytopathology 85:695-698. 1995b.

LUZ, W.C. da. Rizobactérias promotoras de crescimento de plantas e bioproteção. Revisâo Anual de Patologia de Plantas 4:1-47. 1996a.

LUZ, W.C. da. Efeito de rizobactérias promotoras de crescimento de plantas de trigo e de milho. Fitopatologia Brasileira 21:434. 1996b. (Resumo)

LUZ, W.C. da. Espectro de ação de novos fungicidas para tratamento de sementes de milho e seus efeitos no rendimento. Fitopatologia Brasileira 21:369. 1996c. (Resumo)

LUZ, W.C. da. Espectro de ação de fungicidas contra fungos de sementes de milho. $21^{\circ}$ Congresso Nacional de Milho e Sorgo, 4, Londrina, PR. Resumos. 1996d. p.288.

LUZ, W.C. da. Evaluation of seed treatment fungicides for emergence and yield of corn. Fungicide \& Nematicide Tests 52:303. 1997a. (Resumo)

LUZ, W.C. da. Effect of seed treatment on corn pathogen control, stand, and yield. Fungicide \& Nematicide Tests 52:303. 1997b. (Resumo)

LUZ, W.C. da. Efeito de bioprotetores na germinação e no rendimento de grãos de milho. $21^{\circ}$ Congresso Nacional de Milho e Sorgo, 21, Recife, PE, Anais. 1998a. p.227.

LUZ, W.C. da. Efficacy of chemical seed treatments in controlling seed decay of corn. Fungicide \& Nematicide Tests 53:390. 1998b. (Resumo) 


\section{W. C. Luz}

LUZ, W.C. da. Efeito de bioprotetores em patógenos de sementes e na emergência e rendimento de grãos de milho. Fitopatologia Brasileira 26:16-20. 2001a.

LUZ, W.C. da; PEREIRA, L.R. Tratamento de sementes com fungicidas relacionado com o controle de patógenos e rendimento de milho. Ciência Rural 28:537-541. 1998.

LUZ, W.C. da., BERGSTROM, G.C. \& STOCKWELL, C.A. Seed microbiolization for control of Fusarium species in cereais. Phytopathology 87:522. 1997a. (Abstract)

LUZ, W.C. da; BERGSTROM, G.C; \& STOCKWELL, C.A. Seed bioprotectants for control of Fusarium on wheat and corn. Proceedings of the First National Head Blight Forum. St. Paul, Mn, USA. November, 1997b. 